\title{
Gene medicine for cancer treatment: Commercially available medicine and accumulated clinical data in China
}

\author{
Guangyu Ma ${ }^{1,3}$ \\ Hideaki Shimada ${ }^{2}$ \\ Kenzo Hiroshima ${ }^{4}$ \\ Yuji Tada ${ }^{5}$ \\ Nobuo Suzuki ${ }^{3}$ \\ Masatoshi Tagawa' \\ 'Division of Pathology, Chiba Cancer \\ Center Research Institute; ${ }^{2}$ Division \\ of Gastroenterological Surgery, \\ Chiba Cancer Center, Chiba, Japan; \\ ${ }^{3}$ Department of Environmental \\ Biochemistry; ${ }^{4}$ Department of \\ Diagnostic Pathology; ${ }^{5}$ Department \\ of Respirology, Graduate School of \\ Medicine, Chiba University, Chiba, \\ Japan
}

\begin{abstract}
Loss of p53 function compromises genetic homeostasis, which induces deregulated DNA replication, damages DNA, and subsequently results in increased resistance to anticancer agents. Pharmacological approaches using recombinant adenoviruses (Ad) have been developed to restore the p53 functions. Another approach for gene medicine is to modify Ad replication in a tumor-specific manner, which induces tumor cell death without damaging normal tissues in the vicinity. The Ad-derived gene medicines, Ad expressing the wild-type $p 53$ gene and replication-competent Ad defective of the $E 1 B-55 \mathrm{kDa}$ gene, have been tested for their clinical feasibility and became commercially available in China. These agents demonstrated their antitumor activities as a monotherapy and in combination with conventional chemotherapeutic agents. In this article, we summarize the outcomes of clinical trials in China, most of which have been published in domestic Chinese journals, and discuss potential directions of cancer gene therapy with these agents.
\end{abstract}

Keywords: gene therapy, cancer, clinical trials, p53, adenovirus, E1B

\section{Introduction}

Cancer is currently one of the main causes of death in Western and Asian societies. Treatments for advanced-stage cancer are often difficult with a limited efficacy despite multimodal therapeutic strategies. Novel approaches are required to improve the prognosis. A molecular targeting with small-sized synthetic chemicals in a certain type of cancer is currently available and furthermore many candidate molecules are under investigation. Gene therapy is also a possible treatment modality and over the past decades has been investigated preclinically and clinically for their feasibility. According to data published in the Journal of Gene Medicine, there have been 1309 approved clinical trials in the world until July 2007 and $66.5 \%$ of the trials targeted cancer. Historically, a number of clinical trials have been conducted in the Western world but none of the gene medicines have yet been approved by governmental authorities for commercialization. In contrast, adenoviruses (Ad) expressing the human wild-type $p 53$ gene and Ad defective of the E1B-55 kDa molecule, both of which were originally developed and clinically examined for the efficacy in USA, have been admitted in China. The Ad agents have become the first commercially available gene medicine in the world.

In October 2003, the State Food and Drug Administration (SFDA) of China approved type 5 Ad bearing the human wild-type $p 53$ gene (Ad-p53) for the treatment of head and neck cancer. Ad-p53 (Gendicine ${ }^{\circledR}$, Shenzhen SiBiono GeneTech, Shenzhen, China [now incorporated into Benda Pharmaceutical, Wuhan, China]) have been initially developed by Introgen Therapeutics (Advexin ${ }^{\circledR}$, Austin, TX, USA) for head, neck, and lung cancer treatment. While Introgen was working to obtain US 
FDA approval, SiBiono successfully completed clinical studies and launched the product. In November 2005, SFDA also approved type $5 \mathrm{Ad}$ defective of the E1B-55 kDa molecule for head and neck cancer treatment. The E1B-defective Ad were originally developed in USA and were representatively called ONYX-015 (Onyx Pharmaceuticals, Emeryville, CA, USA). While ONYX-015 had been investigated for the clinical efficacy, a Chinese bioventure company, Shanghai Sunway Biotech (Shanghai, China), independently developed E1B-55 kDa-deleted Ad, completed their clinical studies, and commercialized the medicine (Oncorine ${ }^{\circledR}$ ) in 2006. Sunway Biotech has also acquired the exclusive license of ONYX015 in the world.

Since the majority of these clinical results conducted in China has not been well reported in an international conference, many researchers in the Western medical societies have been unaware of the recent progress of cancer gene therapy in China until recently. A large number of cancer patients including Caucasians have received the gene medicine in China and the clinical outcomes were published in major Chinese domestic journals. Many non-Chinese medical scientists were unfortunately unable to access the information. Scientists in the Western countries have recently noticed a successful launching of Gendicine and Oncorine in a Chinese market and come to pay close attention to Chinese clinical data. It is obvious that such commercialization is a remarkable progress for the development of cancer gene therapy but difficulties in accessing such Chinese information were not beneficial for non-Chinese communities as well as the Chinese society. In this review article, we summarize the current status of Ad-p53 and E1B-defective Ad (represented by ONYX-015) and show the clinical data obtained in Chinese medical institutions.

\section{Clinical trials with Ad-p53 Mechanism of the antitumor effects}

Gendicine and Advexin are essentially the same in viral structure. They are recombinant type $5 \mathrm{Ad}$ in which the $\mathrm{E} 1$ region is replaced by either a Rous sarcoma virus (Gendicine) or a cytomegalovirus (Advexin) promoter linked with the human wildtype $p 53$ gene and a poly (A) tail. The Ad-p53 can produce a good amount of $\mathrm{p} 53$ protein in target cells and achieve a variety of cellular responses. The wild-type $\mathrm{p} 53$ plays a central role in maintaining genetic stability in human cells. About a half of human malignancies show loss of $\mathrm{p} 53$ functions, resulting in increased resistance to DNA-damaging agents (Shiraishi et al 2004). Transduction of tumors with Ad-p 53 has demonstrated inhibition of the tumor growth and enhanced the susceptibility to anticancer agents (Blagosklonny et al 1998; Inoue et al 2000; Schuler et al 2001; Sah et al 2003; Schwartzenberg et al 2004; Wang et al 2006). Animal experiments also showed the therapeutic effects to various cancers by way of intratumoral injection and in combination with an anticancer agent or agents. Several lines of the experiments suggested that Ad-p53 produced better antitumor effects to tumors bearing mutated $p 53$ genes than in those with the wild-type $p 53$ gene (Cerrato et al 2001; Eisold et al 2004). Ad are not integrated into host genomes; subsequently a possible risk of secondary Ad-mediated tumorigenesis by activating an oncogene and inactivating a tumor suppressor gene is extremely low and in fact type 5 Ad have not induced tumors in human.

A number of clinical studies demonstrated that intratumoral injection of Ad-p53 resulted in vector-mediated $p 53$ gene expression in various types of tumors and thus produced antitumor activities (Clayman et al 1998; Habib et al 1999; Swisher et al 1999; Singh et al 2002). The clinical trials showed that Ad-p53 administration was safe and well tolerated with minimal adverse reactions to most of the patients. The minimal unfavorable effects exhibited that Ad-p53 had a high therapeutic window and consequently implied that Ad-p53 could be used in combination with other conventional treatments, chemotherapy, and/or radiation therapy. The expressed 553 protein achieved antitumor activities through a number of mechanisms (Muller et al 1998; Nemunaitis et al 2000; Pal et al 2001; Pagliaro et al 2003; Quist et al 2004; Rosenblum et al 2004; Rother et al 2004; Sabine 2004). (a) Induction of apoptotic pathways in tumor cells, which is mediated by a variety of machinery (Muller et al 1998; Bouvard et al 2000; Matsuda et al 2002; Chipuk et al 2004; Leu et al 2004; Taha et al 2004). (b) Blockage of survival signal pathways in tumor cells (Singh et al 2002; Yin et al 2003; Rother et al 2004). (c) Activation of host immune surveillance systems (Yen et al 2000; Cerwenka and Lanier 2003; Rosenblum et al 2004). (d) Down-regulation of the multi-drug resistance genes (Krishna and Mayer 2000), which improves susceptibility of tumor cells to chemotherapy (e) Inhibition of angiogenesis (Dameron et al 1994; Pal et al 2001) and metastasis by suppressing matrix metalloproteinase (Toschi et al 2000; Ala-aho et al 2002; Sun et al 2004).

\section{Clinical results obtained with Gendicine in China}

Several phase II and III clinical trials have been conducted in conjunction with Gendicine and conventional therapies and the combinatorial modality produced better therapeutic benefits than Gendicine alone in most of the studies. 
Gendicine has been currently approved only for head and neck cancer with intratumoral injection and in combination with chemotherapy. A number of clinical studies in fact have been performed with an off-labeled use of Gendicine, which is allowed with permission of individual institutional ethical committees. The agent has been tested as an off-labeled use for lung cancer, breast cancer, mesothelioma, and esophageal, hepatocellular, gastric, colorectal, and pancreatic carcinomas. These studies examined possible additional effects of Gendicine to conventional therapies including hyperthermia. Gendicine has been usually administered intratumorally with $1 \times 10^{12}$ viral particles $(\mathrm{vp})$ irrespective of tumor types. Various administration routes have been investigated including pleural and peritoneal cavity perfusion and even intra-arterial as well as intravenous administration. All the publications mentioned that the most common adverse effect of Gendicine was low-grade fever which was self-cured and suggested that Gendicine was safe and effective. The major concern of the clinical reports is however that the studies did not follow a strict treatment schedule and the selection criteria of patients were ambiguous. Most of the studies involved a relatively small number of the patients and it seems that they were accumulated respective case reports. Nonetheless according to Dr. Peng's presentation at the European Society of Gene and Cell Therapy conference in 2007, more than 7,000 cancer patients including 500 nonresidents in total have received Gendicine and he expected more than 10,000 patients at the end of 2007 . A phase IV study seems to be initiated in China although such information is not well documented.

\section{Head and neck squamous cell carcinoma}

The majority of head and neck squamous cell carcinoma treated with Gendicine (85\%) is nasopharygeal carcinoma
(Chen et al 2003; Zhang et al 2003) because of the high incidence in China. The multicentered trial was conducted with 135 patients, and 77\% were not eligible for surgical resection with stage III or IV and were chemotherapyresistant. The study thereby did not exclude early-staged patients. The protocol was consisted with two therapeutic arms: a combination of Ad-p53 and radiotherapy or radiotherapy alone with the same irradiation doses. In the combination therapy, $1 \times 10^{12} \mathrm{vp}$ Gendicine was administered before the radiotherapy and the tumor responses were evaluated with a computed tomography (CT) or magnetic resonance imaging. The combination therapy demonstrated that the complete remission (CR) and partial response (PR) rate were $64 \%$ and $29 \%$, respectively, whereas the radiation alone group showed that $\mathrm{CR}$ and PR were $19 \%$ and $60 \%$, respectively. The report thereby concluded that Gendicine in combination with radiotherapy produced synergistic effects.

\section{Hepatocellular carcinoma}

Gendicine was tested for 68 and 82 advanced hepatocellular carcinoma patients in combination with chemotherapy and the chemotherapy alone, respectively (Guan et al 2005) (Table 1). Camptothecin, doxorubicin, and 5-fluorouracil were used together with embolization. Gendicine $\left(1-4 \times 10^{12} \mathrm{vp}\right)$ was administered at multiple points into tumor masses under CT guidance every week 48-72 hours after the embolization. Although the precise treatment schedule and clinical features of the patients were not well described, the report mentioned that the response rates of the combination and the control group were $67.6 \%$ and $51.2 \%$, respectively $(p<0.05)$ and the 6 -month survival rate was $76.5 \%$ in the combination and $23.2 \%$ in the control $(\mathrm{p}<0.01)$. The study also reported

Table I Off-label usage with Gendicine in China

\begin{tabular}{|c|c|c|c|c|}
\hline Type of tumor & $\begin{array}{l}\text { Number } \\
\text { of patients }\end{array}$ & Administration routes & $\begin{array}{l}\text { Combination with } \\
\text { other therapies }\end{array}$ & Reference \\
\hline Hepatocellular carcinoma & 68 & $\begin{array}{l}\text { CT-guided intratumor } \\
\text { injection }\end{array}$ & Chemotherapy & Guan et al 2005 \\
\hline \multirow[t]{2}{*}{ Lung cancer } & 15 & CT-guided pneumocentesis & None & Weng et al 2004 \\
\hline & 15 & $\begin{array}{l}\text { CT-guided pneumocentesis } \\
\text { or via bronchial artery }\end{array}$ & Chemotherapy & Guan et al 2005 \\
\hline Soft tissue sarcoma & 12 & Intratumor injection & Hyperthermia & Xiao et al 2007 \\
\hline $\begin{array}{l}\text { Malignant effusions by } \\
\text { malignant mesothelioma } \\
\text { and alimentary tract } \\
\text { carcinoma }\end{array}$ & 32 & Peritoneal injection & Hyperthermia & Liu et al 2006 \\
\hline
\end{tabular}

Abbreviation: $\mathrm{CT}$, computed tomography. 
improvement of quality of patients' life with Gendicine (Guan et al 2005).

\section{Other types of cancer}

Gendicine has been investigated for the efficacy to advanced lung cancer together with and without chemotherapeutic agents (Weng et al 2004) and for soft tissue sarcoma with and without radiotherapy and hyperthermia (Xiao et al 2007). Due to the limited patient numbers, it is difficult to draw any conclusions (Table 1).

\section{Factors involved in Ad-p53-mediated effects}

There are several factors that influence the efficacy of Ad-p53-mediated antitumor effects. One of the major factors is the expression level of type 5 Ad receptors. The receptors of type $5 \mathrm{Ad}$ are consisted of coxsackie adenovirus receptor (CAR) and integrin $\alpha v \beta 3$ and $\alpha v \beta 5$. The expression of $\mathrm{CAR}$, a primary receptor for $\mathrm{Ad}$, is often down-regulated in human tumors, which decreases the transduction efficacy with Ad vectors. Consequently, human tumors with a low CAR expression level showed decreased therapeutic efficiency (Zeimet and Marth 2003). The infection efficiency is also impeded by the presence of Ad-neutralizing antibodies, which are produced by Ad administration irrespective of the injection sites. It is estimated that $60 \%$ or more of the world population, although it depends on area, are positive for antiAd5 antibody prior to the treatment and subsequently even single Ad administration induced high titered neutralizing antibody with ease.

The integrity of signal transduction downstream of the p53 pathways also determines the efficacy. The majority of preclinical studies showed that Ad-p53 produced better therapeutic effects to tumors with mutated $p 53$ genes than those with wild-type $p 53$ gene (Katayose et al 1995; Blagosklonny and el-Deiry 1996; St John et al 2000). Recent clinical studies by Introgen also demonstrated the correlation between the efficacy of Ad-p53 and endogenous p53 gene status and suggest that the status is a biomarker to predict the effectiveness. Expression of p53 restores an appropriate activation of the $\mathrm{p} 53$ downstream signaling in p53-mutated tumor cells probably because p53-mutated cells often have intact p53-downstream pathways. Instead, wild-type p53-bearing tumors cells could have mutated p53downstream cascades, which consequently give resistance to p53-mediated cellular responses despite super-induction of p53. Zeimet and Marth (2003) suggested that tumors with epigenetically deregulated the $p 53$ gene also showed the similar responses to Ad-p53 as found in the tumors with a dominant-negative p53 mutation.

\section{Clinical studies with Ad defective of EIB-55 kDa Development of oncolytic Ad}

Ad-p53 lack the E1 region and are replication-incompetent. The antitumor effects thereby depend on the expression level of transduced p53 and on the integrity in p53-mediated cascades in the target tumors. The other approach to produce Ad-mediated antitumor effects is to use the cytotoxicity that is associated with the viral propagation within cells. Recombinant Ad which can replicate within tumors is a therapeutic strategy because such Ad replication triggers a cytotoxic mechanism different from that by conventional anticancer agents.

Wild-type Ad can replicate efficiently in epithelial cells and are potential cytolytic to tumors. An early clinical trial in the 1950's showed that intratumoral administration of the wild-type Ad of different types induced regression of cervical carcinomas; however, the therapeutic effects were transient. The clinical application of such viruses-mediated cytotoxicity was thereby abandoned due to the lack of sustained clinical efficacy (Smith et al 1956). More recently, recombinant virus technology has led to the development of conditionally replicating Ad that are designed to selectively replicate in tumor cells while leaving normal cells undamaged. An advantage of such replication-competent Ad with an oncolytic property is a continuous spread of the virus progenies released from damaged tumors into neighboring tumor cells even if the initial efficiency of transduction is relatively low. The first generation of such conditionally replicative Ad (also termed as CRAd) was ONYX-015 (d11520 or CI-1042) (Bischoff et al 1996). ONYX-015 is a chimeric Ad consisting of type 2 and type 5 structures with a partial abrogation of the E1B-55 $\mathrm{kDa}$-encoding region, which results in deletion of the protein synthesis. Initially ONYX-015 was considered to efficiently replicate only in cells lacking functional p53 and to induce significant regression of human tumors developed in mice due to the tumor-specific viral replication (Bischoff et al 1996; Rogulski et al 2000). The E1B-55 kDa protein and $\mathrm{E} 4$ orf3 and E4 orf6 proteins binds to p53 and inhibits the p53-mediated apoptosis; subsequently the viral production was enhanced (Yew and Berk 1992). Hypothetically ONYX015 would not be able to prevent p53-induced apoptosis in normal cells and replicate in cells lacking functional 
p53, as found in the majority of human tumors (White 1994; Fulci and Van Meir 1997). Several studies with a limited number of cell lines demonstrated greater cytotoxicity of ONYX-015 to p53-mutated tumors compared to those with the wild-type $p 53$ gene (Bischoff et al 1996). Subsequent studies however denied the correlation between the $p 53$ status and replicative capacity of ONYX-015 (Rothmann et al 1998). Although the precise mechanism of viral replication with respect to the $p 53$ status remains unclear, empirical evidences supported the preferential lysis of tumor cells in comparison with normal cells (Reid et al 2002; Nemunaitis et al 2003).

ONYX-015 has been clinically examined for head and neck cancer and liver metastases of colon cancer with various delivery routes (Reid et al 2002; Nemunaitis et al 2003; Chu et al 2004). Overall, the trials have shown an acceptable safety profile even when the Ad were systemically delivered. The therapeutic efficacy was not however satisfactory when it was used as a single agent but the combinatory use with anticancer agents enhanced the activities. For example, a response rate of ONYX-015 together with cisplatin and 5-fluorouracil, more than 50\% tumor reduction 1 month after the treatment, was $63 \%$ for head and neck cancer whereas that of ONYX-015 and the chemotherapy alone were $14 \%$ and $30 \%-40 \%$, respectively. Despite the promising outcomes, clinical development of ONYX-015 in USA has been discontinued, but not because of medical or scientific issues. Currently the next generations of oncolytic Ad in which the $\mathrm{E} 1$ region is activated by an exogenous transcriptional region and/or E3 region is replaced with a therapeutic transgene such as Escherichia coli cytosine deaminase, an enzyme that can convert 5-fluorocytosine into its toxic metabolite, have been examined for its efficacy and clinical feasibility (Zhan et al 2005). The expression of a transgene in the E3 region is regulated under the control of endogenous viral late gene regulatory elements and linked with the replication of oncolytic Ad (Royds et al 2006).

\section{Clinical application of oncorine in China}

A phase III study of ONYX-015 for head and neck cancer has been suspended in USA but Oncorine (the developmental code name was H101), which is type 5 Ad and essentially the same structure as ONYX-015, had been clinically tested for its efficacy in China. Following phase I and phase II clinical trails which included 191 patients with 13 kinds of carcinoma such as head and neck cancer, breast cancer, and colon cancer, a phase III clinical trail with Oncorine on head and neck cancer was performed with intratumoral injections. It seems that the clinical studies of Oncorine were more convincing than those of Gendicine and the clinical results were comparable to that of ONYX-015 in USA. The maximal tolerance dose was $2 \times 10^{12} \mathrm{vp}$ but lower dose were also effective.

\section{Head and neck squamous cell carcinoma}

$\mathrm{Hu}$ and colleagues (2006) reported that 123 patients were evaluable in a phase III study (Xia et al 2004), which did not report the p53 status of each tumor. The patients received both Oncorine and chemotherapeutic agents; the combination of either cisplatin +5 -fluorouracil or adriamycin +5 -fluorouracil. The study included patients who had not received any chemotherapy before as well as those who were resistant to the chemotherapy. Oncorine was injected intratumorally at the dose of $5 \times 10^{11} \mathrm{vp}$ for 5 consecutive days for a treatment course. The adverse effects were not serious and well-tolerated; fever, local pain at the injection site, and flu-like symptoms were frequently reported. An objective response rate of the group that received both Oncorine and anticancer agents and that of the chemotherapy group as a control were $72.7 \%$ and $40.4 \%$, respectively. The combination therapy was more effective to those who were naïve to the chemotherapy (79.0\% response rate). Oncorine had also been examined for the efficacy with 56 nasopharyngeal carcinoma patients in the combination of chemotherapy (cisplatin +5 -fluorouracil). Oncorine showed better objective response rates in the combination with chemotherapy $(75.6 \%)$ than chemotherapy alone $(57.1 \%)$ but the results were statistically insignificant $(\mathrm{p}=0.081)$. Among the patients who did not have prior chemotherapy, the group of Oncorine with chemotherapy achieved better response rates than that of chemotherapy alone $(86.5 \%$ to $59.4 \%, \mathrm{p}<0.05)$.

Interestingly, the patients who had fever at the time of Oncorine administration had better objective responses than those without fever although statistically not significant. The precise mechanism remains uncharacterized but preclinical data suggest that it was attributable to elevated expression of the heat shock protein, which facilitated late viral RNA export to cytoplasm and subsequently enhanced viral replications (O'Shea et al 2005). The preclinical study also showed that ONYX-015 RNA was poorly transported to cytoplasm in normal cells whereas the transportation was up-regulated in tumors with unknown mechanisms. They suggested that the differential transfer of viral RNA could contribute to the tumor-specific cell lysis with ONYX-015. Another study of trimodal combination of Oncorine, chemotherapy, and radiotherapy was conducted for 56 patients with 
nasopharyngeal carcinoma (Lu et al 2006). Although clinical results of the control group without Oncorine administration were not shown, the trimodal therapy showed $71.4 \%$ of complete responses and $28.6 \%$ of partial responses with minimal adverse effects such as a transient fever and mucosal ulcer in the oral cavity.

\section{Nonsmall cell lung cancer}

A small number of nonsmall cell lung cancer patients have received intratumoral injection of Oncorine $\left(1.5 \times 10^{12} \mathrm{vp}\right)$ together with vinorelbine and cisplatin. In evaluable 19 patients who received Oncorine and the chemotherapy, 5 and 10 patients showed partial response and stable diseases, respectively, while 3 and 9 cases for partial response and stable diseases in 17 patients with the chemotherapy alone. Likewise, one year survival rate and median time to progression were not significantly different between the two groups. Intratumoral injection of Oncrine was safe but the efficacy was not as great as in the case of head and neck cancer (Zhou et al 2006).

\section{Clinical feasibility of ONYX-0I5 in combination treatment}

Heterogenous or decreased CAR expression in tumors also hampered ONYX-015-mediated antitumor effects as observed in the case of Ad-p53 (Zeimet and Marth 2003). In addition, many kinds of tumor cells did not support viral replication of ONYX-015 irrespective of the p53 status. Recent studies however shed light on a heat shock protein which seems to differentially regulate the viral replication between tumors and normal cells (Huang et al 2003; Wang et al 2005). Although the role of thermal effects in the preferential viral replication in tumors is not well characterized, ONYX-015-resistant cells are sensitized by the induction of the heat shock protein (Hu et al 2006) and interestingly, the clinical data implied better clinical outcomes in the patients with a mild fever after ONYX-015 administration. Heat shock proteins are commonly up-regulated in tumor cells and could explain the preferential cytotoxicity of ONYX-015 to tumors. Induction of heat shock responses by pharmacological agents such as benzoquinoid ansamycins or by local hyperthermia can improve the efficiency and is a possible combinatory strategy with ONYX-015.

The trial with hyperthermia was started 1 day after the first viral injection. The injected tumor sites were warmed with a radio-frequency heating instrument for 5 days. The outcomes are not reported due to a limited patient number but the research group raised a possibility of enhanced clinical benefits. Heat shock proteins have influence on antigen presentation in which the proteins bind putative tumor antigens. Increased antigenicity through class I and II of major histocompatibility complexes favors activation of $\mathrm{CD}^{+}$and $\mathrm{CD}^{+} \mathrm{T}$ cells and subsequently up-regulates cell-mediated immunity.

Combination with chemotherapy showed better therapeutic effects but anticancer agents themselves can inhibit the viral proliferation. Several agents are now clinically in use with Oncorine but careful evaluation will be required for clinical outcomes and the viral replications. Multiple injection of Ad generates anti-Ad responses which could be beneficial to prevent systemic viral spread but inhibitory to viral spread into tumors in the vicinity.

\section{Conclusions}

A number of clinical trials have been conducted mainly in USA; however, none of the agents are commercially available in the Western markets. There are several reasons for the delayed governmental approval. During the approval processing, two kinds of medicine, which are originally developed in bioventure companies in USA, have been approved in China; however, the clinical studies from China in general were not adequately organized judged by the standards of Western medical societies. These reports are sometimes fragmentary and merely summarize respective case reports. In particular the studies often did not include a well-controlled group and long-term survival data. There is also a major difference in the clinical outcomes between the Chinese and Western studies, in which the Chinese studies showed better therapeutic effects with the same Ad vectors. One of the reasons is the differential eligibility criteria; Chinese studies sometimes included early staged patients who should be treated with a standard conventional therapy. Secondly, Chinese studies often use the gene medicine in particular in Ad-p53 cases as an adjuvant to the standard therapy. It is not wise at this moment to directly compare the clinical outcomes since several factors are not the same among the studies.

The commercialization nevertheless has given an opportunity for medical scientists to assess the potential clinical benefits of the gene medicine. In addition, it has also enabled an off-labeled use of the gene medicine in China with permission from an individual institutional ethical committee. These current situations lead us to re-evaluate the clinical data obtained in China. All the data indicated that the gene medicine were safe with minimal adverse reactions and seems to be effective in combination with other therapies. 
Ad-p53 can be used in combination with current standard protocols. More than 7,000 cancer patients have received Ad-p53 and many of them were inoffensively treated with a combinatory use of radiotherapy and/or chemotherapy. The treatments with Oncorine in China have been also conducted with the combination and the clinical outcomes are similar as those performed with ONYX-015 in USA. Clinical trials conducted in both countries with Ad-p53 or Ad defective of E1B$55 \mathrm{kDa}$ gene showed that the gene therapy were safe and could be beneficial for the cases in which conventional anticancer treatments have failed; however, many kinds of concerns and medical questions regarding Ad-p53 and Oncorine administration in the clinical settings remain unanswered. Several issues such as limited applicability of the administration due to the topological localization are also clinically important. Currently the Ad are injected intratumorally with good tolerability and moderate clinical activity, while it would be better to deliver intravenously for various types of advanced cancers. Targeting to cancer with specificity is also one of the directions to be investigated. Cancer- and/or tissue-specific modifications of viral coat proteins which can change the vector tropism will increase the selectivity to cancer and consequently reduce the toxicity. Similarly, a new generation of replicative Ad vectors, placing the expression of therapeutic genes under the control of tumor-specific promoters or transcriptional control regions could be a strategy and in fact several trials are also conducted (Deweese et al 2001).

A number of clinical trials for cancer have been planned with replication-incompetent and -competent Ad. Clinical data from Chinese studies provides us a number of suggestions and implications, which would be useful for future clinical studies. In particular, growing numbers of the patients who received the gene medicine in various hospitals in China increase the necessity for phase IV studies. The Chinese clinical studies, when completed at an international standard level, must be enormously valuable in orienting a future direction of gene medicine. On the other hand, the director of SFDA was sentenced for bribery to exercise his power over approval of new medicines in China in 2007. It raised a serious concern in the domestic and the international medical and pharmacological societies. Although the Chinese gene medicine companies declared that they were not involved in the corruption, the approval process of gene medicines in China will be more strictly controlled and monitored in future.

\section{Acknowledgments}

This work was supported by Grants-in-aid for Center of Excellent (COE) Program of the Japanese Ministry of Education, Culture, Sports, Science and Technology, grant-in-aid for scientific research from Japan Society for the Promotion of Science, the grant-in-aid for cancer research (18-10) from the Ministry of Health, Labour and Welfare, and grant-in-aid from the Nichias Corporation and the Futaba Electronics Memorial Foundation.

\section{References}

Ala-aho R, Grenman R, Seth P, et al. 2002. Adenoviral delivery of p53 gene suppresses expression of collagenase-3 (MMP-13) in squamous carcinoma cells. Oncogene, 21:1187-95.

Bischoff J, Kirn D, Williams A, et al. 1996. An adenovirus mutant that replicates selectively in p53 deficient human tumor cells. Science, 274:373-6.

Blagosklonny MV, EL-Deiry WS. 1996. In vitro evaluation of a p53-expressing adenovirus as an anti-cancer drug. Int J Cancer, 67:386-92.

Blagosklonny MV, Giannakakou P, Wojtowicz M, et al. 1998. Effects of p53-expressing adenovirus on the chemosensitivity and differentiation of anaplastic thyroid cancer cells. J Clin Endocrinol Metab, 83:2516-22.

Bouvard V, Zaitchouk T, Vacher M, et al. 2000. Tissue and cell-specific expression of the $\mathrm{p} 53$ target genes: bax, fas, $\mathrm{mdm} 2$ and waf1/p21, before and following ionizing irradiation in mice. Oncogene, 19:649-60.

Cerrato JA, Yung WK, Liu TJ. 2001. Introduction of mutant p53 into a wild-type p53-expressing glioma cell line confers sensitivity to Ad-p53-induced apoptosis. Neuro Oncol, 3:113-22.

Cerwenka A, Lanier LL. 2003. NKG2D ligands: Unconventional MHC class I-like molecules exploited by viruses and cancer. Tissue Antigens, 61:335-43.

Chen C, Pan J, Xu L. 2003. Recombinant adenovirus p53 agent injection combince with radiotherapy in treatment of nasopharyngeal carcinoma: a phase II clinical trial. Zhonghua Yi Xue Za Zhi, 23:2033-5.

Chipuk JE, Kuwana T, Bouchier-H, et al. 2004. Direct activation of Bax by p53 mediates mitochondrial membrane permeabilization and apoptosis. Science, 303:1010-14.

Chu RL, Post DE, Khuri FR, et al. 2004. Use of replicating oncolytic adenoviruses in combination therapy for cancer. Clin Cancer Res, 10:5299-312.

Clayman GL, EL-Naggar AK, Lippman, et al. 1998. Adenovirus-mediated p53 gene transfer in patients with advanced recurrent head and neck squamous cell carcinoma. J Clin Oncol, 16:2221-32.

Dameron KM, Volpert OV, Tainsky MA, et al. 1994. Control of angiogenesis in fibroblasts by 553 regulation of thrombospondin-1. Science, 265:1582-4.

Deweese TL, Vander PH, Li S, et al. 2001. A phase I trial of CV706, a replication-competent, PSA selective oncolytic adenovirus, for the treatment of locally recurrent prostate cancer following radiation therapy. Cancer Res, 61:7464-72.

Eisold S, Linnebacher M, Ryschich E, et al. 2004. The effect of adenovirus expressing wild-type p53 on 5-fluorouracil chemosensitivity is related to p53 status in pancreatic cancer cell lines. World J Gastroenterol, 10:3583-9.

Fulci G, Ishii N, Van Meir EG. 1998. p53 and brain tumors: from gene mutations to gene therapy. Brain Pathol, 8:599-613.

Guan YS, Sun L, Zhou XP, et al. 2005. Combination therapy with recombinant adenovirus-p53 injection (rAd-p53) via transcatheter hepatic arterial chemoembolization for advanced hepatic carcinoma. Shijie Huaren Xiaohua Zazhi, 13:125-7.

Habib NA, Hodgson HJ, Lemoine N, et al. 1999. A phase I/II study of hepatic artery infusion with wtp53-CMV-Ad in metastatic malignant liver tumors. Hum Gene Ther, 10:2019-34.

Huang XF, Ren W, Rollins L, et al. 2003. A broadly applicable personalized heat shock protein-mediated oncolytic tumor vaccine. Cancer Res, 63:7321-9. 
Hu F, Hu X, Yu P, et al. 2006. Abscopal effect on metastatic tumor induced by oncolytic virus of H101 combining with local heating. Ai Zheng, 25:919-24.

Inoue A, Narumi K, Matsubara N, et al. 2000. Administration of wild-type p53 adenoviral vector synergistically enhances the cytotoxicity of anticancer drugs in human lung cancer cells irrespective of the status of p53 gene. Cancer Lett, 157:105-12.

Katayose D, Gudas J, Nguyen H, et al. 1995. Cytotoxic effects of adenovirus-mediated wild-type p53 protein expression in normal and tumor mammary epithelial cells. Clin Cancer Res, 1:889-97.

Krishna R, Mayer LD. 2000. Multidrug resistance (MDR) in cancer: Mechanisms, reversal using modulators of MDR and the role of MDR modulators in influencing the pharmacokinetics of anticancer drugs. Eur J Pharm Sci, 11:265-83.

Leu J, Dumont P, Hafey M, et al. 2004. Mitochondrial p53 activates Bak and causes disruption of a Bak-Mcl1 complex. Nat Cell Biol, 6:443-50.

Lu Y, Hu X, Li F, et al. 2006. Radiotherapy combined with chemotherapy and intratumoral injection of EIB gene-deleted adenovirus (H101) for nasopharyngeal carcinoma. Zhong Liu, 26:453-5.

Matsuda K, Yoshida K, Taya Y, et al. 2002. p53AIP1 regulates the mitochondrial apoptotic pathway. Cancer Res, 62:2883-9.

Muller M, Wilder S, Bannasch D, et al. 1998. p53 activates the CD95 (APO-1/Fas) gene in response to DNA damage by anticancer drugs. J Exp Med, 188:2033-45.

Nemunaitis J, Swisher SG, Timmons T, et al. 2000. Adenovirus-mediated p53 gene transfer in sequence with cisplatin to tumors of patients with non-small-cell lung cancer. J Clin Oncol, 18:609-22.

Nemunaitis J, Cunningham C, Tong AW, et al. 2003. Pilot trial of intravenous infusion of a replication selective adenovirus (ONYX-015) in combination with chemotherapy or IL-2 treatment in refractory cancer patients. Cancer Gene Ther, 10:341-52.

O'Shea CC, Soria C, Bagus B, et al. 2005. Heat shock phenocopies E1B$55 \mathrm{~K}$ late functions and selectively sensitizes refractory tumor cells to ONYX-015 oncolytic viral therapy. Cancer Cell, 8:61-74.

Pagliaro LC, Keyhani A, Williams D, et al. 2003. Repeated intravesical instillations of an adenoviral vector in patients with locally advanced bladder cancer: A phase I study of p53 gene therapy. J Clin Oncol, 21:2247-53.

Pal S, Datta K, Mukhopadhyay D. 2001. Central role of p53 on regulation of vascular permeability factor/vascular endothelial growth factor (VPF/VEGF) expression in mammary carcinoma. Cancer Res, 61:6952-7.

Quist SR, Wang-Gohrke S, Kohler T, et al. 2004. Cooperative effect of adenoviral p53 gene therapy and standard chemotherapy in ovarian cancer cells independent of the endogenous p53 status. Cancer Gene Ther, 11:547-54.

Reid T, Galanis E, Abbruzzese J, et al. 2002. Hepatic arterial infusion of a replicationselective oncolytic adenovirus (d1520): Phase II viral, immunologic, and clinical endpoints. Cancer Res, 62:6070-9.

Rogulski KR, Freytag SO, Zhang K, et al. 2000. In vivo antitumor activity of ONYX-015 is influenced by radiotherapy. Cancer Res, 60:1193-6.

Rosenblum MD, Olasz E, Woodliff JE, et al. 2004. CD200 is a novel p53-target gene involved in apoptosis-associated immune tolerance. Blood, 103:2691-8.

Rother K, Johne C, Spiesbach K, et al. 2004. Identification of Tcf-4 as a transcriptional target of p53 signalling. Oncogene, 23:3376-84.

Rothmann T, Hengstermann A, Whitaker NJ, et al. 1998. Replication of ONYX-015, a potential anticancer adenovirus, is independent of p53 status in tumor cells. J Virol, 72:9470-8.

Royds JA, Hibma M, Dix BR, et al. 2006. p53 promotes adenoviral replication and increases late viral gene expression. Oncogene, 25:1509-20.

Sabine L. 2004. Can China bring its own pipeline to the market? Nat Biotechnol, 22:1497-9.

Sah NK, Munshi A, Nishikawa T, et al. 2003. Adenovirus-mediated wild type $\mathrm{p} 53$ radiosensitizes human tumor cells by suppressing DNA repair capacity. Mol Cancer Ther, 2:1223-31.
Schuler M, Herrman R, DE Greve JL, et al. 2001. Adenovirus-mediated wild-type p53 gene transfer in patients receiving chemotherapy for advanced non-small-cell lung cancer: Results of a multicenter phase II study. J Clin Oncol, 19:1750-8.

Schwartzenberg-bar-Yoseph F, Armoni M, Karnieli E, 2004. The tumor suppressor p53 down-regulates glucose transporters GLUT1 and GLUT4 gene expression. Cancer Res, 64:2627-33.

Shiraishi K, Kato S, Han SY, et al. 2004. Isolation of temperaturesensitive p53 mutations from a comprehensive missense mutation library. J Biol Chem, 279:348-55.

Singh B, Reddy PG, Goberdhan A, et al. 2002. p53 regulates cell survival by inhibiting PIK3CA in squamous cell carcinomas. Genes Dev, 16:984-93.

Smith RR, Huebner RJ, Rowe WP, et al. 1956. Studies on the use of viruses in the treatment of carcinoma of the cervix. Cancer, 9:1211-8.

St John LS, Sauter ER, Herlyn M, et al. 2000. Endogenous p53 gene status predicts the response of human squamous cell carcinomas to wild-type p53. Cancer Gene Ther, 7:749-56.

Sun Y, Zeng XR, Wenger L, et al. 2004. p53 down-regulates matrix metalloproteinase- 1 by targeting the communications between AP-1 and the basal transcription complex. J Cell Biochem, 92:258-69.

Swisher SG, Roth JA, Nemunaitis J, et al. 1999. Adenovirus-mediated p53 gene transfer in advanced non-small-cell lung cancer. J Natl Cancer Inst, 91:763-71.

Taha TA, Osta W, Kozhaya L, et al. 2004. Down-regulation of sphingosine kinase-1 by DNA damage: Dependence on proteases and p53. J Biol Chem, 279:20546-54

Toschi E, Rota R, Antonini A, et al. 2000. Wild-type p53 gene transfer inhibits invasion and reduces matrix metalloproteinase-2 levels in p53mutated human melanoma cells. J Invest Dermatol, 114:1188-94.

Wang H, Qian H, Yu J, et al. 2006. Administration of PUMA adenovirus increases the sensitivity of esophageal cancer cells to anticancer drugs. Cancer Biol Ther, 5:380-5.

Wang XY, Li Y, Yang G, et al. 2005. Current ideas about applications of heat shock proteins in vaccine design and immunotherapy. Int J Hyperthermia, 21:717-22.

Weng Z, Qin TL, Tan SY, et al. 2004. Clinical trial for the treatment of advanced lung cancer by intratumoral injection of rAd p53. Shenzhen Zhong Xi Yi Jie He Za Zhi, 14:206-10.

White E. 1994. Tumour biology: p53, guardian of Rb. Nature, 371:21-2.

Xia ZJ, Chang JH, Zhang L, et al. 2004. Phase III randomized clinical trial of intratumoral injection of E1B gene-deleted adenovirus (H101) combined with cisplatin-based chemotherapy in treating squamous cell cancer of head and neck or esophagus. Ai Zheng, 23:1666-70.

Xiao S, Liu C, Sun Y, et al. 2007. Clinical observation of the effect of recombinant adenovirus- p53 plus radio-thermotherapy on soft tissue sarcoma. Zhong Guo Zhong Liu Lin Chuang, 34:65-7.

Yen $\mathrm{N}$, Ioannides $\mathrm{CG}, \mathrm{Xu} \mathrm{K}$, et al. 2000. Cellular and humoral immune responses to adenovirus and $\mathrm{p} 53$ protein antigens in patients following intratumoral injection of an adenovirus vector expressing wildtype P53 (Ad-p53). Cancer Gene Ther, 7:530-6.

Yew PR, Berk AJ, 1992. Inhibition of p53 transactivation required for transformation by adenovirus early $1 \mathrm{~B}$ protein. Nature, 357:82-5.

Yin Y, Liu YX, Jin YJ, et al. 2003. PAC1 phosphatase is a transcription target of p53 in signalling apoptosis and growth suppression. Nature, 422:527-31

Zeimet AG, Marth C. 2003. Why did p53 gene therapy fail in ovarian cancer? Lancet Oncol, 4:415-22.

Zhan J, Gao Y, Wang W, et al. 2005. Tumor-specific intravenous gene delivery using oncolytic adenoviruses. Cancer Gene Ther, 12:19-25.

Zhang S, Xiao S, Liu C, et al. 2003. Treatment of head and neck squamous cell carcinoma by recombinant adenovirus-p53 combined with radiotherapy: a phase II clinical trial of 42 cases. Zhonghua Yi Xue Za Zhi, 23:2023-8.

Zhou C, Xu Y, Ni J, et al. 2006. Oncolytic adenovirus H101 in combination with vinorelbine/ cisplatin chemotherapy for advanced non-small cell lung cancer. Zhong Liu, 26:613-17. 